\title{
Bandwidth Broadening of MEMS Vibration Energy Harvesters by Voltage-boost Rectifier Circuit
}

\author{
Yukiya Tohyama, ${ }^{*}$ Hiroaki Honma,${ }^{1}$ Hiroshi Toshiyoshi, ${ }^{1}$ and Daisuke Yamane ${ }^{2 * *}$ \\ ${ }^{1}$ Institute of Industrial Science, The University of Tokyo, 4-6-1 Komaba, Meguro, Tokyo 153-8505, Japan \\ ${ }^{2}$ Department of Mechanical Engineering, Ritsumeikan University, \\ 1-1-1 Noji-Higashi, Kusatsu, Shiga 525-8577, Japan
}

(Received July 2, 2021; accepted October 29, 2021)

Keywords: MEMS, vibration, energy harvester, voltage boost, rectifier

This paper presents a methodology to extend the working bandwidth of vibration energy harvesters (VEHs) by using a voltage-boost rectifier (VBR) circuit. The VBR circuit rectifies small ac voltages of MEMS VEHs and boosts them to a dc voltage sufficient for the operation of the subsequent circuitry. The proposed system using the VBR circuit can work regardless of the VEH type. Also, the VBR circuit can be monolithically integrated with CMOS LSI without using other external components. The developed VBR circuit is experimentally evaluated using a MEMS VEH and successfully delivers boosted dc voltages even when the input ac amplitude is below $0.4 \mathrm{~V}$ in the frequency range $(<1 \mathrm{kHz})$ of environmental vibrations. We confirm that the working bandwidth can be increased by employing the VBR circuit compared with the use of conventional diode rectification.

\section{Introduction}

In IoT systems, miniaturized massive wireless sensor nodes are key interface elements of the physical and cyber worlds. Energy harvesters can be an energy source for these sensor nodes, where battery replacement is difficult. Among several energy harvesting methods, utilizing vibration energy is promising because environmental vibrations ubiquitously exist even at night or indoors. $^{(1)}$

MEMS technology has enabled us to downscale vibration energy harvesters (VEHs), and the output power of VEHs has increased in recent years. ${ }^{(2-6)}$ Most VEHs generate the maximum output power under their resonance conditions, where the working frequencies are bound to their resonance frequencies. The amplitude of the output signal suddenly decreases when the working frequencies, in other words, the frequencies of the input vibration, deviate from the resonance frequency. Thus, to overcome this issue, many works have been reported on the bandwidth enhancement of VEHs.

The broadband techniques are categorized into mechanical and electrical approaches. The mechanical approaches include linear and nonlinear mechanisms that require additional

\footnotetext{
*Corresponding author: e-mail: tohyama@iis.u-tokyo.ac.jp

** Corresponding author: e-mail: dyamane@fc.ritsumei.ac.jp

https://doi.org/10.18494/SAM.2022.3502
} 
mechanical elements, which would increase the total device size or constrain device design flexibility, as seen in arrayed devices, combined eigenmodes, multistable configurations, amplitude-limiting systems, ${ }^{(6-10)}$ and so forth. The conventional electrical approaches that utilize nonlinear electrical interface circuits $^{(11-14)}$ require the use of off-chip discrete components and complicated circuit architectures. ${ }^{(15)}$

In this paper, we present a broadband technique for VEHs realized by adopting a voltageboost rectifier (VBR) as an electrical interface circuit. Unlike conventional electrical interface circuits for increasing the bandwidth, the VBR can be monolithically integrated with CMOS LSI without using other external components, such as off-chip inductors. Moreover, the system can be used regardless of the VEH type. In Sect. 2, the methodology is briefly described, and in Sect. 3, the experimental results are presented.

The concept of the proposed technique has already been presented in our previous work. ${ }^{(16)}$ In this paper, we newly evaluate the frequency and voltage ranges of the VBR. The efficiency of the VBR is also discussed.

\section{System Overview}

The concept of the proposed system is shown in Fig. 1. The output port of a VEH is connected to the input of a VBR circuit, and the output dc voltage of the VBR is delivered to the subsequent load circuits or storage components. We aim to design the VBR threshold voltage to be lower than those of diode rectifiers and the VBR output voltage to be higher than those of diode rectifiers. As shown in the voltage-frequency characteristic curves of the VEH output and input ports in Fig. 1, the available bandwidth around the resonance frequency of the VEH becomes wider when the threshold voltage becomes lower. The $V_{D D}$ level in Fig. 1 shows the dc voltage required for the subsequent circuits. In the case of conventional diode rectification, the

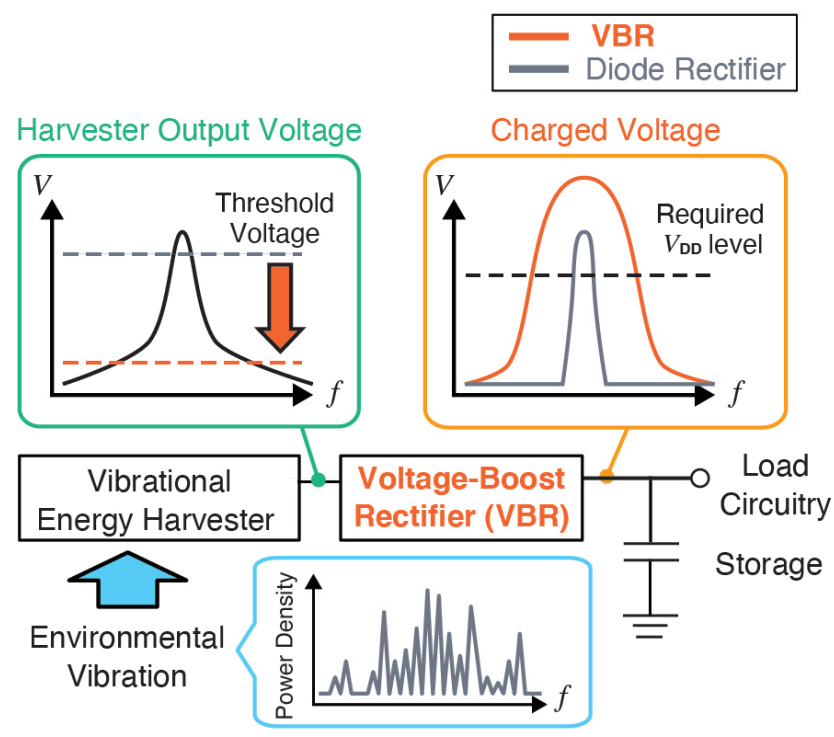

Fig. 1. (Color online) Concept of bandwidth extension system using VBR. 
output voltage of VEHs should be higher than the sum of $V_{D D}$ and the voltage drop across the diodes. Thus, the practical threshold voltage for the VEH diode rectification can be higher than the threshold voltage of diodes. The use of $\mathrm{dc}-\mathrm{dc}$ boost converters after the diode rectification increases the overall system size because off-chip inductors are needed. The VBR presented in this work can be developed by the standard CMOS processes and thus be monolithically integrated with other peripheral circuits, which is useful for miniaturization and packaging.

Figure 2 shows a schematic image of the proposed energy harvesting system using a VBR circuit and a MEMS VEH. We designed a single-ended VBR based on a Dickson-type charge pump topology. ${ }^{(17)}$ The ac input $\left(V_{i n}\right)$ and the ground node are alternately connected to the pumping nodes through coupling capacitors $(C)$. The dc output voltage $\left(V_{\text {out }}\right)$ with $N+1$ $\mathrm{N}$-channel metal-oxide-semiconductor field-effect transistors (NMOS-FETs) can be expressed as ${ }^{(18)}$

$$
V_{\text {out }}=N\left(\frac{C}{C+C_{P}} V_{A C}-\frac{I}{f\left(C+C_{P}\right)}-V_{T H}\right)-V_{T H},
$$

where $C_{P}$ is the parasitic capacitance at each pumping node, $V_{A C}$ the peak-to-peak voltage of the ac input signal, $I$ the average current at the output load, $f$ the ac signal frequency, and $V_{T H}$ the threshold voltage of the NMOS-FETs. Thus, when $V_{\text {out }}$ becomes positive, then the following condition is required:

$$
V_{A C}>\frac{C+C_{P}}{C} \frac{N+1}{N} V_{T H}+\frac{I}{C f}
$$

This provides a minimum ac input voltage level. What is different from conventional diode rectifiers is that as long as the ac input voltage becomes higher than the threshold voltage defined by Eq. (2), we can design the boosted dc output voltage by tuning the circuit parameters such as $C$ and $N$. Such VBRs have been employed for RF energy harvesters with antennae that receive

VBR

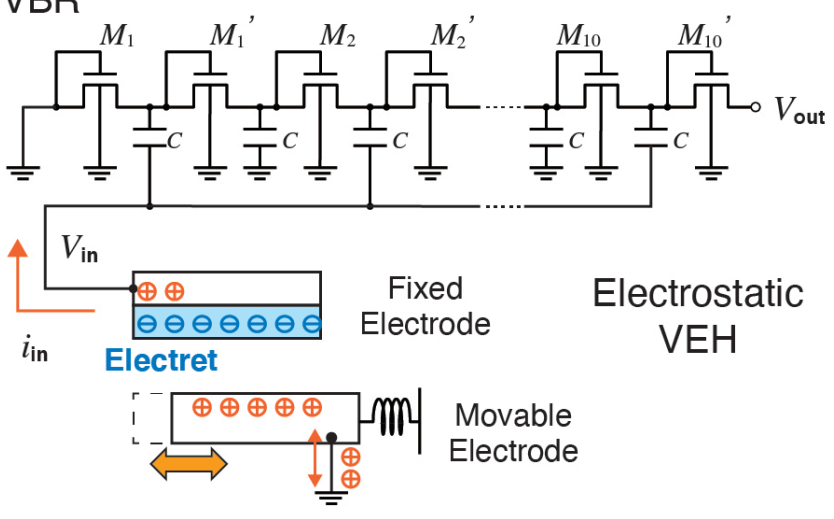

Fig. 2. (Color online) Schematic of VBR circuit and MEMS VEH. 
unused radio waves propagating in the surrounding environment and convert them to electrical current as alternative power sources for batteries. ${ }^{(19)}$ In RF energy harvesters, the input signal levels are relatively lower than those of VEHs, ${ }^{(20)}$ and the RF signal frequency $(\mathrm{MHz}-\mathrm{GHz})^{(19)}$ is considerably higher than the frequencies of the environmental vibrations $(<1 \mathrm{kHz})^{(1,21,22)}$ used for energy harvesting. Recent works indicate that VBRs have potential as a broadband technique for VEHs. ${ }^{(16,23)}$

In this work, we designed a VBR for an electrostatic VEH reported elsewhere ${ }^{(24)}$ and evaluated the performance using both simulation and experimental results to confirm the effect of bandwidth expansion. As shown in Fig. 2, a pair of electrodes is used. One of the electrodes is fixed and moves along the harvester's package frame. The other electrode is suspended by springs and moves relative to the fixed electrode according to the inertial forces of input vibrations. The change in the electrical potential of the electrostatic VEH drives the VBR as one of the VEH electrodes is connected to the input of the VBR. The minimum operating frequency was previously designed to be below $100 \mathrm{~Hz}$ to meet the requirement of environmental vibration harvesting. ${ }^{(1,21,22)}$ As a proof-of-concept system, we designed a VBR circuit within a footprint of $1.2 \times 0.9 \mathrm{~mm}^{2}$ and developed it using a $0.7 \mu \mathrm{m}$ Si CMOS technology. The coupling capacitor was designed to be $1 \mathrm{pF}$, and 10 pumping stages were integrated by using 20 sets of NMOS-FETs. The output dc voltage was designed to be higher than $1 \mathrm{~V}$ even when the frequency of the input vibration becomes the minimum operation frequency, as VEHs are assumed to be power sources for low-power electronics. ${ }^{(25)}$ The typical $V_{T H}$ of the NMOS-FET used was around $0.6 \mathrm{~V}$, and we optimized the circuit parameters to make the minimum input ac level as close to $V_{T H}$ as possible. Accordingly, the minimum input ac amplitude was designed to be lower than $1 \mathrm{~V}$ while maintaining the output dc level higher than $1 \mathrm{~V}$, which cannot be realized by conventional rectifiers with non-negligible voltage drops.

Figure 3 shows the power conversion efficiency of the VBR calculated by LTspice (Analog Devices Inc.). The VBR was excited by the sinusoidal input voltage at $100 \mathrm{~Hz}$ whose amplitude was changed from 0.8 to $1.6 \mathrm{~V}_{\mathrm{pp}}$. A $100 \mathrm{pF}$ capacitance is connected to the output port of the VEH. The instantaneous value of the ratio between the output power and input power is

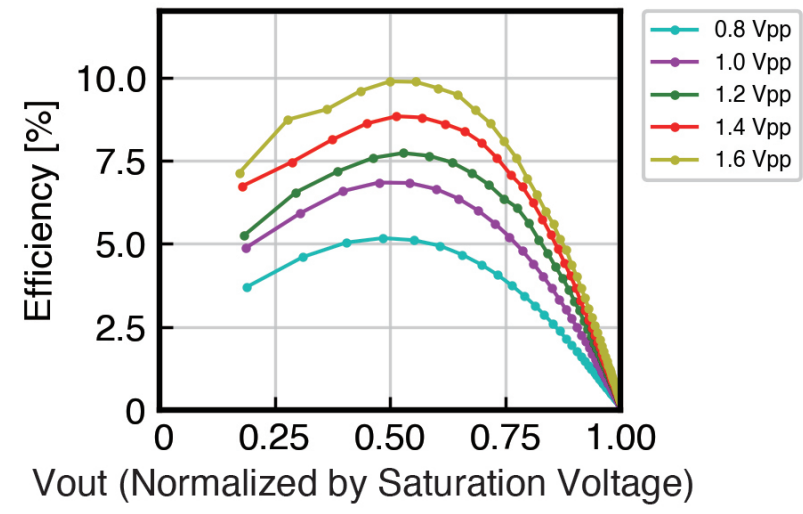

Fig. 3. (Color online) Simulation results of the instantaneous value of power conversion efficiency as a function of output voltage normalized by saturation value. The amplitude of the input voltage is changed as a parameter. 
calculated as a function of $V_{\text {out }}$ from $0 \mathrm{~V}$ to the saturation voltage. Input and output power are derived as the products of voltages and currents measured at the input and output terminals of the VBR. Each curve has a peak at half the saturation voltage, and the efficiency increases with increasing input voltage. A previously reported efficiency ${ }^{(17)}$ was $15 \%$ (after correction for the identical topology for the charge pump circuit), which is in the same order as our results. The efficiency of VBRs is mainly dependent on the number of stages $N$ and the transistor size, namely, the gate width $W$ and the gate length $L .{ }^{(26)}$ The main factor limiting the efficiency is $W$ because the proposed VBR adopted $0.7 \mu \mathrm{m}$ CMOS technology while the previous circuit adopted $90 \mathrm{~nm}$ CMOS technology.

\section{Evaluation Results}

Figure 4(a) shows the experimental setup for the evaluation of the VBR chip alone. The VBR circuit was developed on a die of $1.5 \times 1.5 \mathrm{~mm}^{2}$ area, and wire bonded and sealed on a printed circuit board for evaluation [Fig. 4(b)]. As illustrated in Fig. 4(a), a function generator (Agilent

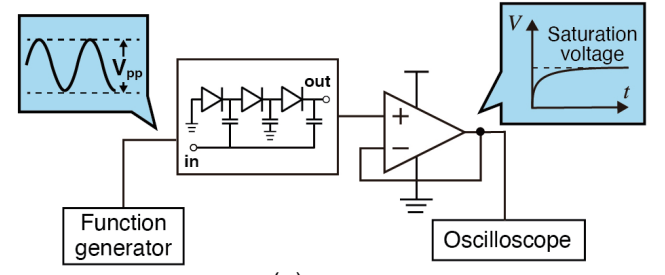

(a)

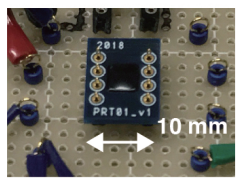

(b)

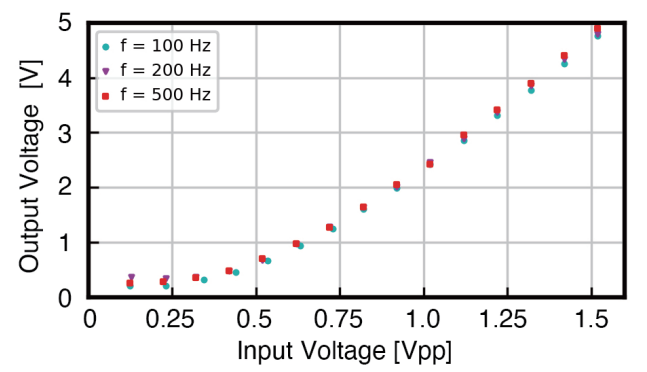

(c)

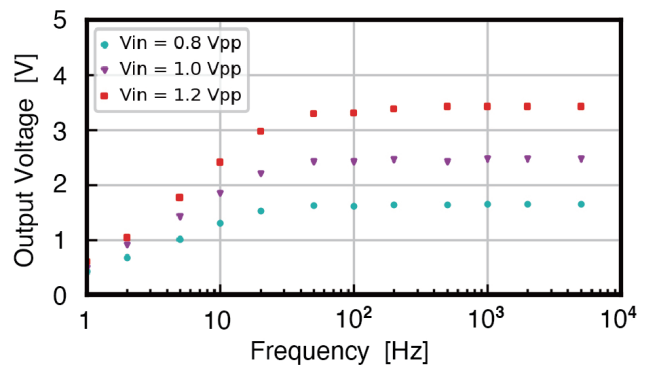

(d)

Fig. 4. (Color online) Evaluation of single characteristics of VBR chip. (a) Experimental setup, (b) image of VBR chip, (c) output voltage as a function of input voltage amplitude and (d) as a function of input voltage frequency. 
Technologies, 33220A) was used to apply sinusoidal voltage to the input port of the VBR circuit. We measured the VBR output signal by an oscilloscope (Agilent Technologies, DSO1014A) with a buffer circuit.

Figure 4(c) shows the measurement results of the boosted dc voltage of the VBR as a function of the input ac level where peak-to-peak input ac voltages at frequencies of 100,200, and $500 \mathrm{~Hz}$ were used. The frequency range below $500 \mathrm{~Hz}$ was designed according to the characteristics of various environmental vibration sources. ${ }^{(1)}$ As shown in Fig. 4(c), we confirmed that the output dc voltage was higher than $1 \mathrm{~V}$ even when the input ac amplitude from the ground level, which was half the peak-to-peak input ac voltage, was below $0.4 \mathrm{~V}$.

Figure 4(d) shows the measured output dc voltage as a function of the input signal frequency where the peak-to-peak input ac voltages are $0.8,1.0$, and $1.2 \mathrm{~V}_{\mathrm{pp}}$. The output de voltage increased with the peak-to-peak input ac voltage. Each output dc voltage saturated below around $100 \mathrm{~Hz}$. This frequency dependence follows Eq. (1), which indicates that discharge by leakage current is compensated at $100 \mathrm{~Hz}$.

Figure 5 illustrates the experimental setup used to evaluate the bandwidth extension characteristics of the VBR circuit. As shown in Fig. 5(a), a shaker (Emic, 9514-AN/SD 373-A, DCS-98S Smart) was used to apply vibrations to a MEMS VEH as reported elsewhere. (24) The output voltage from the MEMS VEH was rectified and boosted to a DC voltage by the VBR circuit. The output voltage of the VBR circuit was measured by an oscilloscope (Agilent Technologies, DSO1014A) through a buffer circuit. Figure 5(b) shows photographs of the MEMS VEH set on the shaker to apply single-axis vibrations and the VBR circuit chip implemented on a printed circuit board.

Figure 6 shows the transient characteristics of the measured voltage over time at $1 \mathrm{mG}(\mathrm{G}=$ $9.8 \mathrm{~m} / \mathrm{s}^{2}$ ) input sinusoidal acceleration. In this experiment, the frequency of acceleration was set to be around the resonant frequency of the MEMS VEH. ${ }^{16)}$ Figure 6(a) presents the measurement results obtained using a single diode rectifier instead of the VBR circuit. The

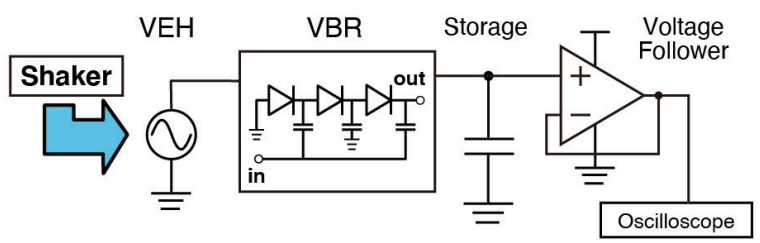

(a)

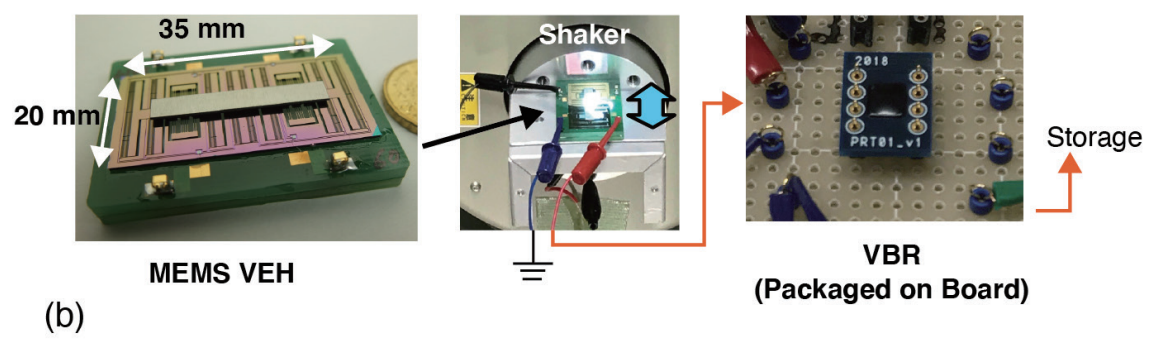

Fig. 5. (Color online) Experimental setup for evaluation of VBR characteristic with MEMS VEH. 
diode was developed in the same Si CMOS manufacturing process and the threshold voltage was around $0.6 \mathrm{~V}$.

The measurement results obtained using the VBR circuit are shown in Fig. 6(b). Comparing Figs. 6(a) and 6(b), it can be seen that the VBR circuit successfully boosted the output voltage, although it required additional charging time to obtain the saturation voltage. This charging time is permissible for intermittent operation. Step-like responses are observed on the voltage curves around $10 \mathrm{~s}$ in Fig. 6(b). A possible cause of these responses is the frequency shift of the VEH. The quality factor of the VEH is 250 under the open circuit condition; ${ }^{(16)}$ therefore, the instability in the resonant frequency of the VEH has caused a change in the output voltage.

Figure 7 shows the saturation voltage spectra with different input sinusoidal accelerations. Figures 7(a)-7(c) represent the measurement results with the input acceleration amplitudes of 1, 5 , and $10 \mathrm{mG}$, respectively. The peak voltage of each spectrum was found to be around the

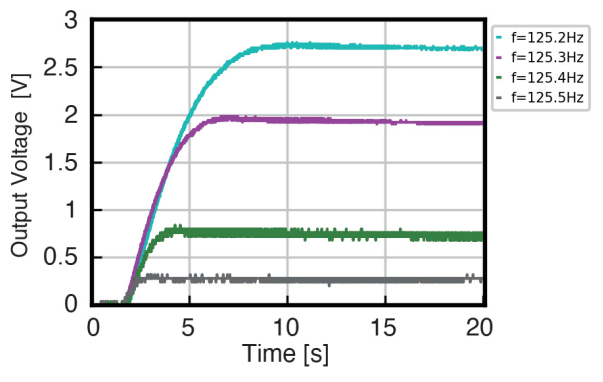

(a)

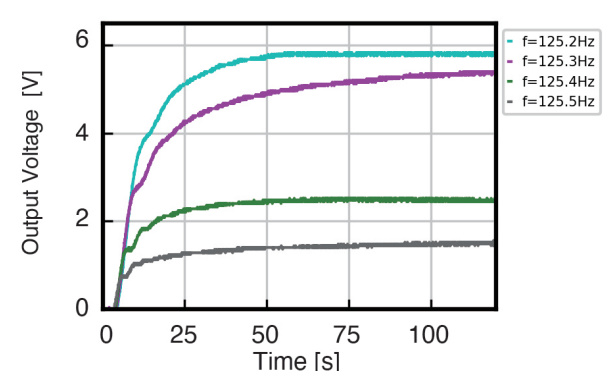

(b)

Fig. 6. (Color online) Transient results of the measured output voltage of (a) diode rectifier and (b) VBR with $1 \mathrm{mG}$ input sinusoidal acceleration.
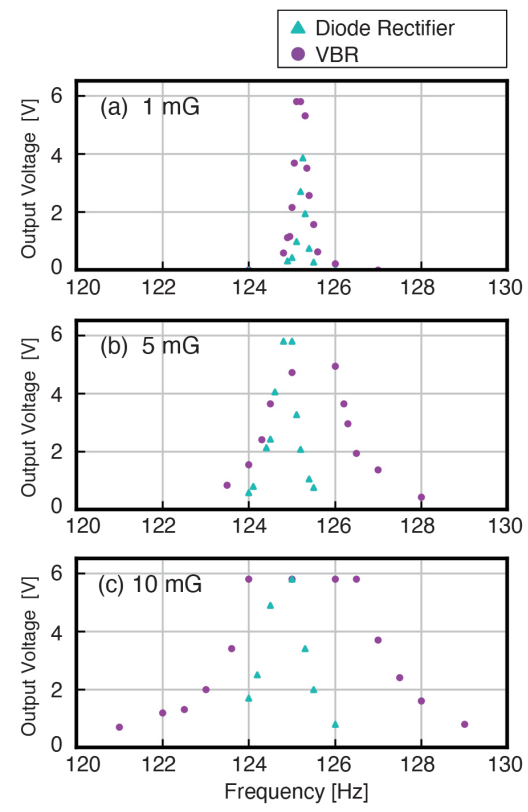

Fig. 7. (Color online) Frequency characteristics of saturation output voltage with input acceleration amplitudes of (a) $1 \mathrm{mG}$, (b) $5 \mathrm{mG}$, and (c) $10 \mathrm{mG}$. 
resonant frequency of the MEMS VEH. In addition to the experiments using the VBR circuit, we also measured the saturation voltage spectra with the use of a single diode rectifier in place of the VBR circuit as shown in Figs. 7(a)-7(c). The saturation characteristics of the measured spectra around the charged voltage of $6 \mathrm{~V}$ were associated with the maximum output voltage of the buffer circuit used. It was confirmed that the working bandwidth can be increased by employing the VBR circuit compared with the use of conventional diode rectification. The enlargement ratio of the bandwidth depends on the original bandwidth of VEHs used, because the output voltage of the VBR is dependent on the frequency and the amplitude of the input voltage.

\section{Conclusions}

We proposed a methodology for the bandwidth extension of MEMS VEHs using a VBR circuit. Unlike conventional electrical interface circuitry for bandwidth enhancement, the VBR circuit can be monolithically integrated with CMOS LSI without using other external components. Also, the system can work regardless of the VEH type, and thus it can be combined with other mechanical bandwidth-broadening methods. The experimental results showed that the developed VBR circuit successfully boosted the dc voltage, even when the input ac amplitude was below $0.4 \mathrm{~V}$, around the frequency level of environmental vibrations. The working bandwidth of the MEMS VEH was increased by using the VBR circuit compared with that using the conventional method with the diode rectifier. These results confirmed that the proposed methodology will be useful for extending the bandwidth of MEMS VEHs.

\section{Acknowledgments}

This work was supported in part by JST PRESTO Grant Number JPMJPR17R9, Japan; JST CREST Grant Numbers JPMJCR15Q4, JPMJCR19Q2, and JPMJCR21Q2, Japan; and JSPS 18H01490, 19K05232, 20J14049, and 21K14509, Japan.

\section{References}

1 S. Roundy, P. K. Wright, and J. Rabaey: Comput. Commun. 26 (2003) 1131. https://doi.org/10.1016/S01403664(02)00248-7

2 S. P. Beeby, M. J. Tudor, and N. M. White: Meas. Sci. Technol. 17 (2006) 175. https://doi.org/10.1088/09570233/17/12/R01

3 S. Saadon and O. Sidek: Energy Convers. Manag. 52 (2011) 500. https://doi.org/10.1016/j.enconman.2010.07.024

4 Y. Suzuki: IEEJ Trans. Electr. Electron. Eng. 6 (2011) 101. https://doi.org/10.1002/tee.20631

5 H. Toshiyoshi, S. Ju, H. Honma, C.-H. Ji, and H. Fujita: Sci. Technol. Adv. Mater. 20 (2019) 124. https://doi.org /10.1080/14686996.2019.1569828

6 Y. Tan, Y. Dong, and X. Wang: J. Microelectromech. Syst. 26 (2017) 1. https://doi.org/10.1109/ JMEMS.2016.2611677

7 J. Twiefel and H. Westermann: J. Intell. Mater. Syst. Struct. 24 (2013) 1291. https://doi. org/10.1177/1045389X13476149

8 R. L. Harne and K. W. Wang: Smart Mater. Struct. 22 (2013) 023001. https://doi.org/10.1088/0964$1726 / 22 / 2 / 023001$

9 H. Liu, C. Lee, T. Kobayashi, C. J. Tay, and C. Quan: Smart Mater. Struct. 21 (2012) 035005. https://doi. org/10.1088/0964-1726/21/3/035005 
10 K. Murotani and Y. Suzuki: J. Micromech. Microeng. 28 (2018) 104001. https://doi.org/10.1088/1361-6439/ aac8cc

11 D. Guyomar, A. Badel, E. Lefeuvre, and C. Richard: IEEE Trans. Ultrason. Ferroelectr. Freq. Control 52 (2005) 584. https://doi.org/10.1109/TUFFC.2005.1428041

12 D. Guyomar and M. Lallart: Micromachines 2 (2011) 274. https://doi.org/10.3390/mi2020274

13 T. Yildirim, M. H. Ghayesh, W. Li, and G. Alici: Renewable Sustainable Energy Rev. 71 (2017) 435, https://doi. org/10.1016/j.rser.2016.12.073

14 F. Dell'Anna, T. Dong, P. Li, Y. Wen, Z. Yang, M. R. Casu, M. Azadmehr, and Y. Berg: IEEE Circuits Syst. Mag. 18 (2018) 27. https://doi.org/10.1109/MCAS.2018.2849262

15 M. R. Sarker, S. Julai, M. F. M. Sabri, S. M. Said, M. M. Islam, and M. Tahir: Sens. Actuators, A 300 (2019) 111634. https://doi.org/10.1016/j.sna.2019.111634

16 Y. Tohyama, H. Honma, N. Ishihara, H. Toshiyoshi, and D. Yamane: Proc. 20th Int. Conf. on Solid-State Sensors, Actuators and Microsystems (Transducers 2019) \& Eurosensors XXXIII (Berlin, 2019) 1463. https:// doi.org/10.1109/TRANSDUCERS.2019.8808622

17 G. Papotto, F. Carrara, and G. Palmisano: IEEE J. Solid-State Circuits 46 (2011) 1985. https://doi.org/10.1109/ JSSC.2011.2157010

18 J. F. Dickson: IEEE. J. Solid-State Circuits 11 (1976) 374. https://doi.org/10.1109/JSSC.1976.1050739

19 M. Cansiz, D. Altinel, and G. K. Kurt: Energy 174 (2019) 292. https://doi.org/10.1016/j.energy.2019.02.100

20 S. Kim, R. Vyas, J. Bito, K. Niotaki, A. Collado, A. Georgiadis, and M. M. Tentzeris: Proc. IEEE 102 (2014) 1649. https://doi.org/10.1109/JPROC.2014.2357031

21 E. K. Reilly, F. Burghardt, R. Fain, and P. Wright: Smart Mater. Struct. 20 (2011) 125006. https://doi. org/10.1088/0964-1726/20/12/125006

22 C. Wei and X. Jing: Renewable Sustainable Energy Rev. 74 (2017) 1. http://dx.doi.org/10.1016/j.rser.2017.01.073

23 Y. Tohyama, H. Honma, N. Ishihara, H. Sekiya, H. Toshiyoshi, and D. Yamane: J. Phys. Conf. Ser. 1407 (2019) 012101. https://doi.org/10.1088/1742-6596/1407/1/012101

24 H. Honma, H. Mitsuya, G. Hashiguchi, H. Fujita, and H. Toshiyoshi: J. Micromech. Microeng. 28 (2018) 064005. https://doi.org/10.1088/1361-6439/aab514

25 F. F. Zulkifli, J. Sampe, M. S. Islam and M. A. Mohamed: Am. J. Appl. Sci. 12 (2015) 335. https://doi. org/10.3844/ajassp.2015.335.344

26 J. Yi, W.-H. Ki, and C.-Y. Tsui: IEEE Trans. Circuits Syst. I Regul. Pap. 54 (2007) 153. https://doi.org/10.1109/ TCSI.2006.887974 